\title{
A DECISION SUPPORT APPROACH TO TRUST MODELING IN NETWORKED ORGANIZATIONS
}

\author{
Nada Lavrač (1, 2), Peter Ljubič (1), Mitja Jermol (1), Stefan Bollhalter (3) \\ (1) Jožef Stefan Institute, Ljubljana, Slovenia \\ (2) Nova Gorica Polytechnic, Nova Gorica, Slovenia \\ (3) Virtuelle Fabrik AG, Arbon, Switzerland
}

\begin{abstract}
The main motivation for organizations to e-collaborate is to enable knowledge and resource sharing in order to effectively solve a business task. Virtual organizations (VOs) can be formed dynamically from a cluster of experts/organizations when a new business opportunity arises. The main strength of a VO lies in the range of competencies the partners are able to offer jointly through collaboration. One of the difficulties in VO creation is appropriate partner selection with mutual trust, as well as the support for trust estimation and monitoring. High trust is a necessary prerequisite for $V O$ creation, knowledge sharing, resource sharing and taking joint risks. This paper presents a decision support approach to modeling trust in a network of collaborating organizations.
\end{abstract}

\section{INTRODUCTION}

The main motivation for organizations to e-collaborate is to harness dispersed expertise, enable knowledge sharing, learning and flexible resource management in order to effectively solve a new business task. This leads to more flexibility, at a cost of increased complexity of a virtual business environment. E-collaboration requires a different set of management processes since collaboration is a fundamentally voluntary process. Collaborative networked organizations are nonstatic (continually evolving) networks enabled by Information and Communications Technology (Camarinha-Matos, 2003; McKenzie and van Winkelen, 2001). Virtual organizations (VOs) are a form of collaborative networked organizations.

The main strength of a virtual organization lies in the range of competencies the partners are able to offer jointly through collaboration. To fully exploit this advantage, problems of efficiently storing, updating, sharing, promoting and transferring knowledge need to be solved. These problems are solved by appropriate knowledge management which - in addition to the technological solutions provided by knowledge technologies - needs to address also organizational, economic, legislative, psychological and cultural issues (McKenzie, 2001).

Virtual organizations (VOs) can be formed dynamically from a cluster of experts/organizations when a business opportunity arises. As a VO is formed to meet a specific demand, it dissolves once the demand is fulfilled. VO creation is a difficult process, requiring the strategic and management decision making processes to be substantially different from those in traditional organizations, where decisions are made at the top and implemented at the bottom. One of the difficulties in VO 
creation is appropriate partner selection with mutual trust, as well as the support for the management of trust. High trust is a necessary prerequisite for knowledge sharing, resource sharing and taking joint risks. It is also one of the most important aspects to be considered in the VO creation process.

Establishing trust is one of the most important mechanisms in collaborative projects, since the possibility of opportunistic behavior of partners cannot be eliminated by formal contracts. In moderately sized VOs of a few dozen partners, means for trust building include personal meetings, regular communication, sharing of information and knowledge, and stable rules of the game, whereas computational means can also be beneficial to support trust modeling. Computational means for trust modeling are, however, indispensable in large VOs in which personal meetings and communication are hindered by the size of the VO.

In order to propose a mechanism appropriate for modeling trust between collaborating partners, the conditions for trust building and management need to be analyzed first. Some of the factors that affect mutual trust and need to be acquired to enable mutual trust estimation include: partner's competencies, reputation and performance in previous collaborations.

This paper proposes a decision support approach to modeling trust in a network of collaborating organizations, aimed at improved trust management in VOs and decision support in the process of creating virtual organizations. In our paper, the term "trust modeling" is used to denote a simple model of trust relations between networked organizations. The model is represented by a graph, where notes denote reputation and arcs indicate collaboration between organizations. Visualization of the graph enables a useful insight of "trust relations" to a VO broker when creating VO for a specific business case.

A trust model of networked organizations is created through following steps:

1. Each organization is given a simple questionnaire in which it estimates experience in collaboration with other organizations.

2. For each questionnaire, a numerical estimate of reputation, collaboration and trust are computed by a multi-attribute decision support system DEXi, for each pair of organizations.

3. A graph of nodes (organizations) and arcs (collaborations) is formed. Size of the node represents the reputation of a partner, whereas the weight of the arc represents the collaboration between the connected partners.

The paper is organized as follows. Section 2 presents a questionnaire-based decision support approach to trust modeling, illustrated by a study of trust modeling in Virtuelle Fabrik, an industrial cluster in the area of mechanical engineering, followed by the lessons learned from this study. Section 3 presents related work, followed by conclusions and plans for further work.

\section{A HIERARCHICAL MULTI-ATTRIBUTE DECISION SUPPORT}

This section present an approach to trust modeling, based on hierarchical multiattribute decision making, applied to modeling trust between partners of an industrial cluster in the area of mechanical engineering. 


\subsection{The proposed approach to trust modeling}

For trust modeling, the decision making problem of trust estimation can be decomposed into decision sub-problems; e.g., a mutual trust estimate can be computed as a weighted sum of values of different decision criteria. This computation can be performed by a utility aggregation functions used in hierarchical multi-attribute decision support systems in which values of top-level decision criteria are computed by aggregating values of decision criteria at lower levels of a hierarchical tree, which can be used to decompose a decision making problem into sub-problems. A hierarchical decomposition of a decision problem into subproblems is shown in Figure 1.

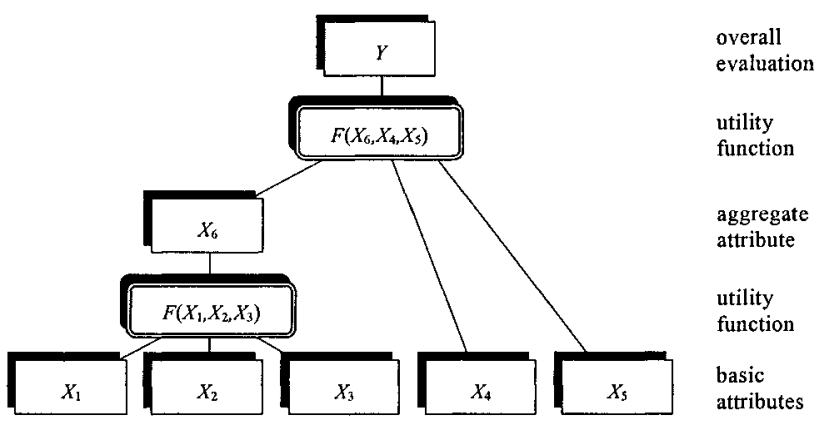

Figure 1 - Components of a multi-attribute decision support model.

Decision support system DEXi, used in our approach to trust modeling, enables the development of qualitative hierarchical decision support models. DEXi is based on the DEX (Bohanec and Rajkovic, 1990) which is used to evaluate incompletely or inaccurately defined decision alternatives, by employing distributions of qualitative values, and evaluating them by methods based on probabilistic or fuzzy propagation of uncertainty. While DEXi is a simpler variant of DEX, lacking some of the DEX functionality, it has better graphical and reporting capabilities, and has therefore been used for decision support in our study.

Knowledge about mutual trust can be acquired through a simple questionnaire that a partner of a networked organization can fill-in to describe the competencies of his own organization and the collaborating partner's performance in previous joint collaborations (for a set of organizations with which the partner has collaborated in past joint projects).

The proposed fields of a questionnaire are listed below:

- partner's own competencies (list of competencies)

- competencies of the collaborating partner (list of competencies), and

- collaborating partner's trust estimate based on: estimated collaborating partner's reputation (image, market share), number of successful joint past collaborations, estimate of the profit made in joint collaborations, estimate of the partner's timeliness in performing assigned tasks, estimate of the partner's quality of performance and products, and estimate of the partner's appropriateness of costs of performance and products. 
A set of questionnaires, filled-in by a partner, results in a star-shaped network: a partner who has filled-in a questionnaire for $N$ collaborating partners is represented by a node, and links to nodes representing collaborating partners. If the questionnaires are filled-in by $M$ partners, this leads to a multi-star network of collaborations in which thick links represent partners with highest mutual trust. In accordance with the link analysis approach to the analysis of social networks (Kleinberg 1988), nodes with most incoming links of high value represent the most trusted partners in the network (the authorities), while the nodes with most outgoing links represent the best connected nodes (the hubs). Hubs and authorities exhibit what could be called a mutually reinforcing relationship, computed through iterative relaxation of the following equations:

$$
\begin{aligned}
& \operatorname{Hub}(p)=\sum_{q: p \rightarrow q} \text { Authority }(q) \\
& \text { Authority }(p)=\sum_{q: q \rightarrow p} \operatorname{Hub}(q)
\end{aligned}
$$

\subsection{The Virtuelle Fabrik trust model: Estimating business reputation and collaboration of VF partners}

The DEXi modeling approach was applied to trust modeling in Virtuelle Fabrik (VF), an industrial cluster of companies in the area of mechanical engineering. In the actual questionnaire, only the questions about each VF partner's reputation and collaborations were taken into the account. The selected multi-attribute decision support model for trust estimation is shown in Figure 2.

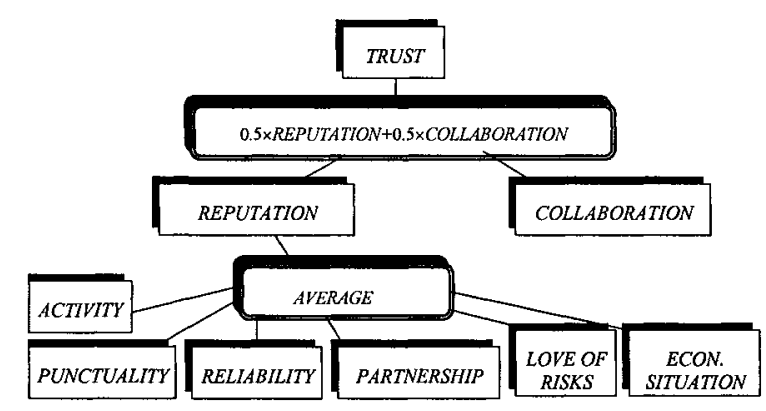

Figure 2 - The selected trust estimation model for Virtuelle Fabrik.

Reputation was modeled through the following VF partner's properties: activity, punctuality, reliability, partnership, love of risk and economical situation. Each of the properties had values from 1 to 6 (1-very bad, 6 - very good), and the overall reputation was computed as the average of values of the basic input attributes. Collaboration between VF partners was evaluated by values 0 to $3(0-$ no collaboration, 3 - strong collaboration), as shown in Table 1. For trust estimate calculation, normalized values of properties are used.

Figure 3 shows the Virtuelle Fabrik trust network. In the visualization tool developed for this application, higher trust can be estimated, when the node representing an organization is wider and/or the connection between two 
collaborating partners is thicker. 'Non-trustful' partners (below the user-defined threshold) do not appear as nodes of the trust network. Specifically, in Figure 3, reputation estimates are depicted by the size of nodes representing individual companies, and the collaborations estimates by the width of arcs between two collaborating companies.

Table 1 - Information about companies (left-hand side table) and their cooperation (right-hand side table). Missing values in the property table are ignored in the model, while, missing values cooperation matrix denote zero values (no collaboration), which are omitted for clarity only.

\begin{tabular}{|c|c|c|c|c|c|c|c|c|c|c|c|c|c|c|c|}
\hline & & 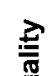 & $\geq$ & $\frac{2}{\frac{2}{n}}$ & $\frac{y}{0}$ & 茜 & \multicolumn{9}{|c|}{ cooperation matrix } \\
\hline 므 & 惫 & క气 & $\frac{\underline{0}}{\mathrm{\omega}}$ & $\frac{\text { ty }}{\pi}$ & 흐 & d & id & 1 & 2 & 3 & 4 & 5 & 6 & 7 & 8 \\
\hline 1 & 2 & 5 & 6 & 4 & & 5 & 1 & & & & 3 & & & 1 & \\
\hline 2 & 4 & 5 & 6 & 5 & 3 & 5 & 2 & & & & & & & & 1 \\
\hline 3 & 2 & 5 & 6 & 5 & 4 & 5 & 3 & & & & & & 1 & & \\
\hline 4 & 5 & 6 & 6 & 6 & 6 & 6 & 4 & 3 & & & & & 2 & 2 & \\
\hline 5 & 5 & 5 & 6 & 6 & 5 & 6 & 5 & & & & & & & 1 & \\
\hline 6 & 2 & 5 & 6 & 4 & 4 & 6 & 6 & & & 1 & 2 & & & & \\
\hline 7 & 5 & 5 & 6 & 6 & 6 & 4 & 7 & 1 & & & 2 & 1 & & & \\
\hline$\ldots$ & & & & & & & $\ldots$ & & & & & & & & \\
\hline
\end{tabular}

\subsection{The lessons learned}

In the Virtuelle Fabrik trust modeling application the following lessons were learned.

- The developed decision support approach and trust visualization tool are useful for VF trust estimation.

- The graph developed by the visualization mechanism shows an accurate picture of the status of Virtuelle Fabrik and its potential VO configurations.

- Some known partner relationships were confirmed.

- Data collection has to be done carefully, as it is a very sensitive issue. There is a certain offending potential of collecting trust modelling data and graphically depicting partner relationships.

- Despite a potential danger of offending non-active and non-connected partners, the approach can also have the opposite, motivating effect: non-active partners may explain the reasons for their situation and the desire for becoming more active members of the network. 


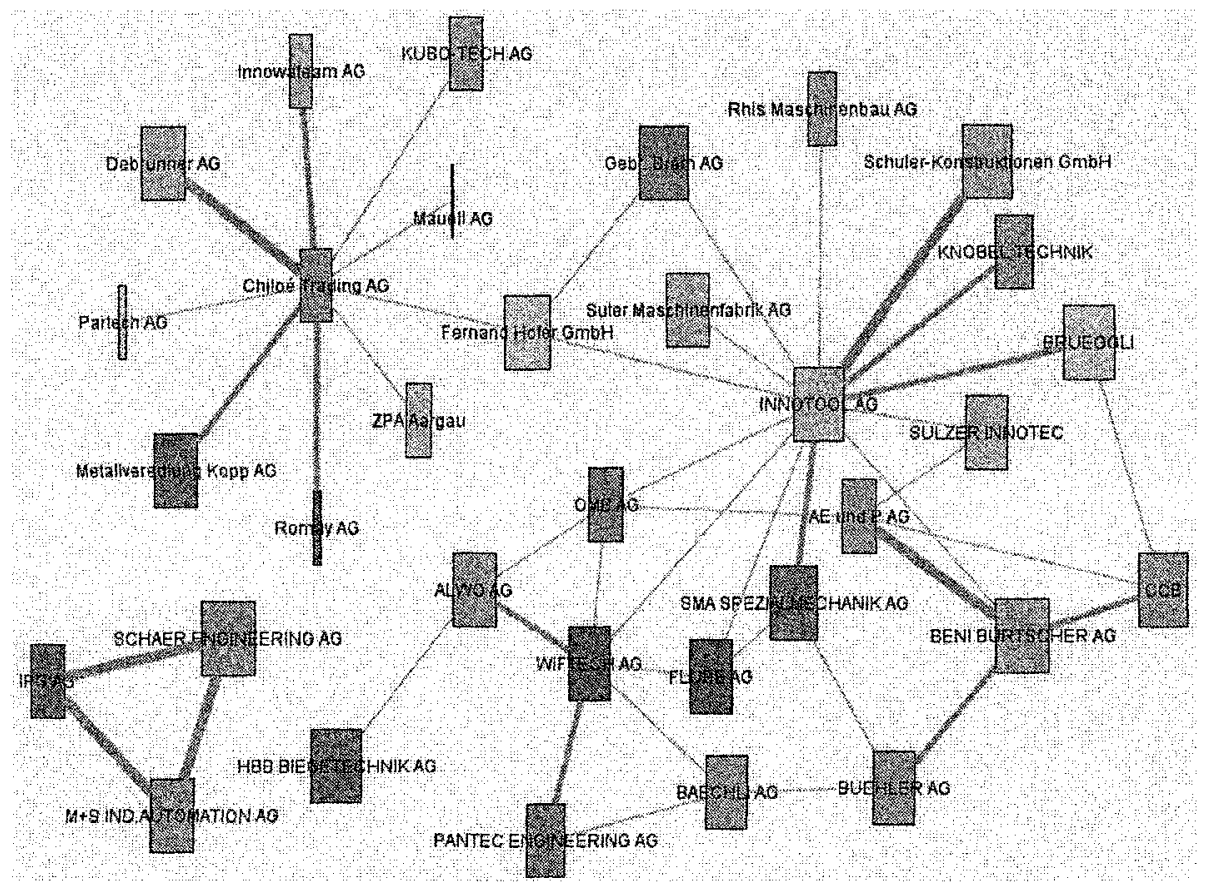

Figure 3 - Visualization of the Virtuelle Fabrik trust network, with node labels corresponding to company names. The figure shows he best connected subset of organizations of the Virtuelle Fabrik industry cluster.

\section{RELATED WORK}

Recent applications of knowledge technologies show their potential for improved trust modeling, knowledge and competence mapping, competence directory formation, partner selection and matching, partner and competency search. Managing trust for the Semantic Web has been of particular interest of researchers [Richardson et al., 2003; Domingos and Richardson, 2001]. Some of these approaches can be used for trust modeling aimed at facilitating the partner selection process and the matching of competencies for a new business opportunity.

Of special significance to our current approach of evaluating trust (using a utility function) is the work of Richardson et al. [2003]. Drawing the analogy from the semantic web, different organizations in a network can be viewed as information sources (of varying qualities) and the competencies of each organization can be treated in a similar way to those of contents on the semantic web in the form of logical assertions or statements. For each organization, which is directly linked to its partner, trust between them can be computed using a utility function like the one proposed in Section 2. Every organization also has a certain belief in its own statements asserted (which are basically its competencies). The belief of an organization in its partner's competencies is then computed and is used as a basic criterion for selecting partners when a new business opportunity arises. 
Social network analysis aimed at modeling network phenomena is an established research field [Wasserman and Faust, 1998; Barabási, 2002]. In addition, trust modeling research (see links to research on trust and reputation at http://moloko.itc.it/trustmetricswiki/moin.cgi/AnalyzedTrustMetrics) has also gained much attention. There has recently been a flood of networking tools made available to individuals and organizations. Selected approaches are described in some more detail below. Other approaches (e.g., accessible at www.zerodegrees.com, center.spoke.com) can also be checked.

LinkedIn (http://www.linkedin.com) is the premier provider of online networking tools, currently used to network over 1.7 million professionals all across the globe. By managing their existing professional networks on LinkedIn, professionals can discover new inside connections and reach business people through referrals from people they know and trust. LinkedIn facilitates hiring, business partnerships and access to industry experts through referrals based on strong and mutually confirmed connections.

Knowmentum (http://www.itsnotwhatyouknow.com) is a tool that supports business, career, and social networking. It is intended to be used by motivated individuals world-wide. CEOs, directors, executives, department managers and sales persons, as well as students, alumni and college associations use Knowmentum to establish communications between job seekers and available positions.

Ryze (http://www.ryze.com) helps people make connections and grow their own networks, with the intention to increase their businesses, build careers, find jobs and make sales. They can also join special networks related to their industry, interests or location. More than 200 organizations host networks on Ryze to help their members interact with each other and enable the growth of their organizations.

\section{CONCLUSIONS AND FURTHER WORK}

The proposed approaches to trust modeling can be used for several purposes, such as ranking of partners according to their research reputation, joint collaborations and the overall trust estimate, visualization of the entire trust network, and finding wellconnected sub-graphs with high trust utility value, representing 'cliques' of partners with strong mutual trust.

Using the proposed approaches for ranking, visualization of the trust network and cliques of partners is relatively straightforward. Implementation of other functions - such as finding the best partners with respect to their competencies and mutual trust, request the computation of the utility function based on the match between the requested expertise with the partners' competencies, and the overall mutual trust of the selected partner sub-graph. This functionality will be developed in further work. One of the outcomes of this trust modelling study is the design of a future awareness raising platform for Virtuelle Fabrik. The main function of the future Automatic Collaboration Trigger (ACT) tool is the activation of VF partners, based on on-line information collection, securing permanent awareness of collaborative business opportunities. The development of the ACT platform is planned in future work. 


\section{Acknowledgments}

The support of the Slovenian Ministry of Higher Education, Science and Technology programme Knowledge Technologies (2004-2008) and the European Integrated Project ECOLEAD (European Collaborative Networked Organizations Leadership Initiative, 2004-2007) are acknowledged.

\section{REFERENCES}

1. Barabási, A. L. Linked. The new science of networks. Cambridge: Perseus Publishing, 2002.

2. Bohanec, M. and Rajkovix, V. DEX: An expert system shell for decision support, Sistemica, Vol. 1, No. 1, 145-157, 1990.

3. Camarinha-Matos, L.M. and Afsarmanesh H. Elements of a base VE infrastructure. J. Computers in Industry, Vol. 51, No. 2, 139-163, 2003

4. Domingos, P. and Richardson, M. Mining the network value of customers. In Proceedings of the 7 th International Conference on Knowledge Discovery and Data Mining, 57-66, 2001. San Francisco.

5. Grobelnik, M. and Mladenić, D. Analysis of a database of research projects using text mining and link analysis. In Mladenić D, Lavrać N, Bohanec M, and Moyle S. (editors) Data Mining and Decision Support: Integration and Collaboration, 157-167, Kluwer, 2003.

6. Kleinberg

7. McKenzie J. and van Winkelen C. Exploring E-collaboration Space. Henley Knowledge Management Forum, 2001..

8. Richardson, M., Agrawal, R. and Domingos, P. Trust management for the Semantic Web. In Proceedings of the 2nd International Semantic Web Conference, 351-368, 2003. Sanibel Island.

9. Wasserman, S. and Faust, K.: Social Network Analysis. Methods and applications. USA: Cambridge University Press, 1998.

10. Kleinberg, J. Authoritative sources in a hyperlinked environment. In Proc. 9th ACMSIAM Symposium on Discrete Algorithms, 1998. 